\title{
Naphthalenes in Neogene sediments of the Shinjo basin, Japan
}

\author{
Akira Shimoyama, Masayosi Hagiwara and Shinya Nomoto \\ Department of Chemistry, University of Tsukuba, Tsukuba 305-8571, Japan
}

(Received December 20, 1999; Accepted April 12, 2000)

\begin{abstract}
Naphthalene and its methyl homologs were found in Miocene to Pliocene sediments of the Shinjo basin. The total concentrations of naphthalenes ranged from 26 to $5,700 \mathrm{ng} \mathrm{g}^{-1}$ from the top to the bottom sediments of an about 1,600 m thick sequence. Naphthalenes were mainly distributed in the Kusanagi Formation and their depth profile showed a characteristic feature, indicating a concentration peak at the onset of oil-generation layer. The ratio of 2- to 1-methylnaphthalene as well as the relative abundance of dimethylnaphthalene isomers, except for 1,8-isomer, showed no depth dependence. Therefore, it seems that no significant isomerizations of the naphthalene homologs had occurred during the burial diagenesis around and above the oil-generating zone in the sediments. However, as to the least stable 1,8dimethylnaphthalene isomer, the negative logarithmic change of the relative abundance of the isomer (Alexander et al., 1984) was observed showing a trend of increasing with depth.
\end{abstract}

\section{INTRODUCTION}

Many investigations have been reported on naphthalene and its alkyl homologs found in sediments and petroleum. Relative distributions of alkylnaphthalene isomers have been used to obtain information about the thermal maturity of sedimentary rocks, coals and crude oils (Radke et al., 1982a, 1984, 1994; Alexander et al., 1985). Naphthalenes are known to be widespread constituents of geological materials and believed to have been derived partly from nonaromatic natural precursors such as terpenoids (Chaffee et al., 1984; Hayatsu et al., 1987; Püttman and Villar, 1987; Strachan et al., 1988). Increasing maturity generally increases the ratio of structurally more stable $\beta$ - to less stable $\alpha$-substituted isomers. Alexander et al. (1984) reported that the relative abundance of 1,8-dimethylnaphthalene was a sensitive indicator of thermal maturity of sediments, though the concentrations of this least stable isomer are usually low and determined less accurately than those of the other isomers. The relative abundances of the other dimethylnaphthalene isomers have been reported to show only moderate correlation with vitrinite reflectance in the range be- low $1.5 \%$ (Radke et al., 1984). Therefore, it could be possible to consider maturity levels of sedimentary organic materials by examination of isomer distribution patterns of methylated naphthalenes in sediments.

In the present study, we determined naphthalenes in the Neogene Shinjo sediments and found a characteristic molecular distribution in the Kusanagi Formation. In addition, we describe the evaluation of the distribution patterns of methyland dimethylnaphthalene isomers.

\section{EXPERIMENTAL}

\section{Samples}

Sediment samples analyzed in this study were collected at outcrops along the Oashizawa route in the Shinjo basin of Yamagata Prefecture, northeastern Japan, in 1980. These samples were portions of common samples for the joint study of organic and inorganic constituents and their diagenesis (Taguchi et al., 1986). They are chiefly marine shales and mudstones of the Nakawatari and Noguchi Formations of Pliocene and of the Furukuchi and Kusanagi Formations of Late to Middle Miocene, in stratigraphically descending 
order. They were numbered discontinuously from 83110 to 90105 of the $1600 \mathrm{~m}$ thick sedimentary sequence. Further information about the stratigraphic and lithologic sections of these formations was described in Taguchi et al. (1986).

Approximately $100 \mathrm{~g}$ each of freshly powdered samples had been prepared from hand sized specimens of the common samples. Portions of each had already been used for the studies of carbon contents and stable carbon isotopic compositions of extractable organic compounds and kerogen (Shimoyama and Matsubaya, 1985), nitrogenous bases (Shimoyama et al., 1988), monocarboxylic acids and $\gamma$-lactones (Shimoyama et al., 1991), and dicarboxylic acids (Mita et al., 1996). In this examination, we analyzed twelve sediment samples for naphthalenes.

\section{Analyses}

For each analysis, $1.0 \mathrm{~g}$ of powdered sample was extracted at room temperature with $3 \mathrm{ml}$ benzene/methanol $(4: 1, \mathrm{v} / \mathrm{v})$ mixture by sonication for $30 \mathrm{~min}$. After centrifugation, the supernatant was saved. The extraction and centrifugation processes were repeated ten times and the saved supernatants were combined. The combined solution was concentrated to about $1 \mathrm{ml}$ under a stream of nitro- gen, before separation by silica gel column chromatography. $n$-Hexane was used for the elution of saturated hydrocarbons, benzene for the aromatic hydrocarbons, and methanol for the heteroatom-containing compounds. The aromatic fraction was concentrated in a stream of nitrogen taking care to avoid complete evaporation and the volume of the concentrated solution was adjusted $50 \mu 1$.

The identification and quantification of individual components in the aromatic fraction were carried out by a gas chromatograph (GC) coupled to a mass spectrometer (MS) (Shimadzu GCMS QP-1000A). The GC was equipped with a fused silica capillary column $(60 \mathrm{~m} \times 0.25 \mathrm{~mm}$ I.D. $)$ coated with TC-1701 (GL Sciences Inc.) and helium was used as a carrier gas. The oven temperature was programmed to give $90^{\circ} \mathrm{C}$ for initial 1 min, from 90 to $130^{\circ} \mathrm{C}$ at a rate of $10^{\circ} \mathrm{C} \mathrm{min}{ }^{-1}$, from 130 to $210^{\circ} \mathrm{C}$ at $2{ }^{\circ} \mathrm{C} \mathrm{min}{ }^{-1}$, and from 130 to $250^{\circ} \mathrm{C}$ at $4^{\circ} \mathrm{C} \min ^{-1}$. The MS was scanned every $1.0 \mathrm{~s}$ over $\mathrm{m} / \mathrm{z} 45-300$ in the electron impact ionization mode at $70 \mathrm{eV}$ with the ion source temperature at $250^{\circ} \mathrm{C}$. The spectral data obtained were stored in the GCMS QP-1000A computer. To avoid laboratory contamination, all glassware was preheated at $500^{\circ} \mathrm{C}$ for at least $3 \mathrm{~h}$ and organic

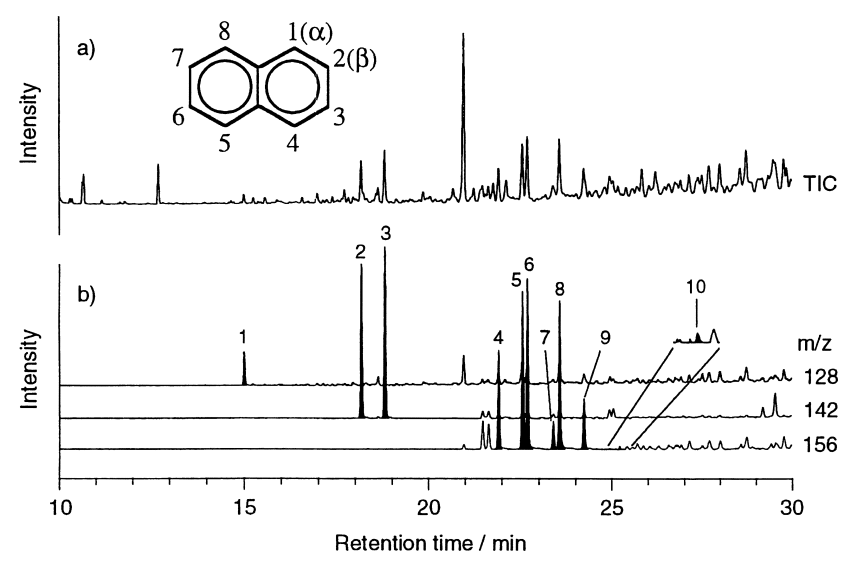

Fig. 1. Gas chromatograms of naphthalenes in the Shinjo-90101. a) Total ion chromatogram. b) Mass fragmentograms. Peak No., 1; naphthalene, 2; 2-methylnaphthalene, 3; 1-methylnaphthalene, 4; 2,6- and 2,7dimethylnaphthalene, 5; 1,3- and 1,7-dimethylnaphthalene, 6; 1,6-dimethylnaphthalene, 7; 1,4dimethylnaphthalene, 8; 1,5- and 2,3-dimethylnaphthalene, 9; 1,2-dimethylnaphthalene, 10;1,8dimethylnaphthalene. 
solvents were doubly distilled.

Compounds were identified by comparison of retention times on the GC and of MS fragment patterns with those of standard compounds. Abundances of naphthalenes were estimated by peak areas on mass fragmentograms of molecular ions. All the standard compounds were used for naphthalene, monomethylnaphthalenes and dimethylnaphthalenes. Standard compounds were purchased from Aldrich Chemical Comp., Inc., Tokyo Kasei Kogyo Co., Ltd., and Wako Pure Chemical Industries, Ltd.

\section{RESUlTS AND DISCUSSION}

\section{Concentrations}

Typical mass fragmentograms of m/z 128, 142, and 156 together with a total ion chromatogram are shown in Fig. 1. The peaks of naphthalene $(\mathrm{m} / \mathrm{z} 128)$, two isomers of methylnaphthalene (m/z 142), 1,2-, 1,4-, 1,6- and 1,8-dimethylnaphthalene $(\mathrm{m} / \mathrm{z} 156)$ were completely resolved each other. However, co-elution of the 1,3- and 1,7-, 1,5- and 2,3-, and 2,6- and 2,7-dimethylnaphthalene isomer pairs occurred and determination of individual abundances of these isomers could not be carried out. Table 1 gives the concentrations of these naphthalenes in the sediment samples. The most abundant compounds, methylnaphthalenes, were present at the level of $1000 \mathrm{ng} \mathrm{g}^{-1}$ in the sample 83003. The thermodynamically least stable 1,8 -isomer among the dimethylnaphthalene isomers (Smith and Batts, 1992) was detected at the level ranging from 0.2 to $11 \mathrm{ng} \mathrm{g}^{-1}$ in all the samples, while concentrations of the other dimethylnaphthalene isomers were about one or two orders of magnitude larger. Concentrations of the isomers other than 1,8-dimethylnaphthalene varied more greatly depending on the samples.

Since several investigators pointed out that the volatility of dinuclear aromatics makes them more susceptible to evaporation losses during separation and analysis than those of tri- or tetranuclear aromatics (Radke et al., 1982b; Alexander et al., 1985), we carried out a test experiment to estimate the recovery of naphthalenes during an

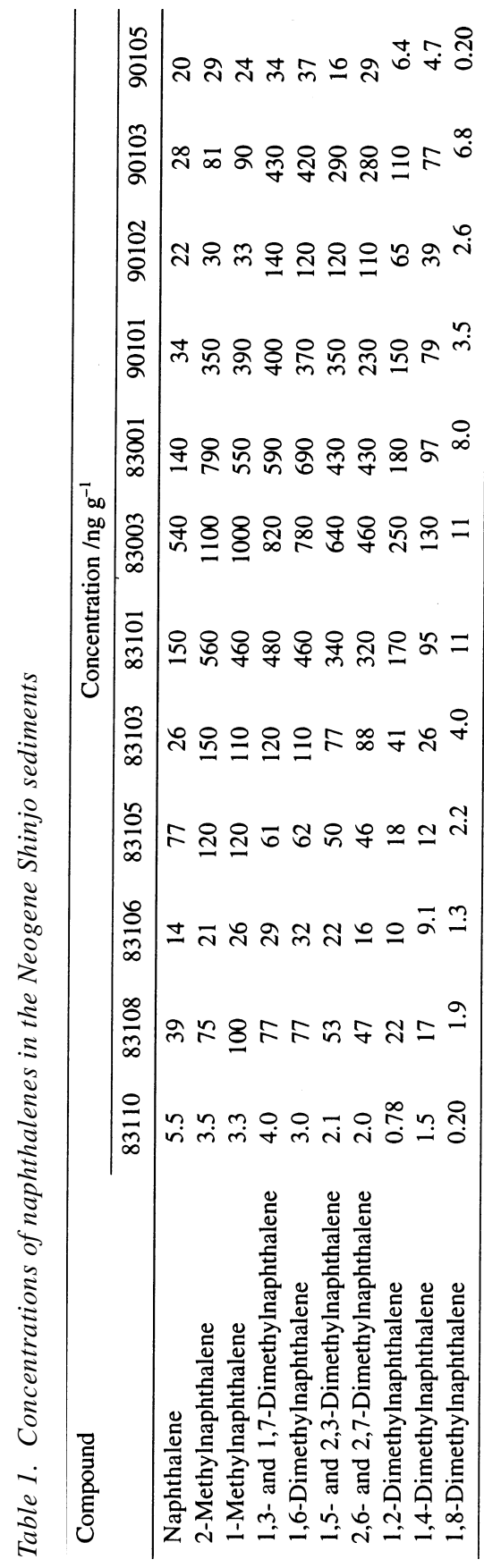


evaporation process. A solution of naphthalene, methylnaphthalenes and seven isomers of dimethylnaphthalene together with pyrene, which is virtually nonvolatile under the concentration conditions and can be used as an internal standard, was concentrated under the same conditions as we employed in the analysis of the sediments. The recovery of each compound after evaporation was evaluated by gas-chromatographic analysis. The ratios of recovered naphthalenes to that of pyrene were from 0.92 to 0.95 . The evaporation loss could be minimized in our concentration method, in which a nitrogen stream is gently blown over the solution below a room temperature and at atmospheric pressure with special precaution to avoid evaporation to dryness.

\section{Depth profile}

The concentrations of naphthalene, methylnaphthalenes and dimethylnaphthalenes at the 12 sediment horizons over the stratigraphic sequence are plotted in Fig. 2, with the contents of the total organic carbon (TOC) and the extractable organic compounds (EOC) (Shimoyama and Matsubaya, 1985).

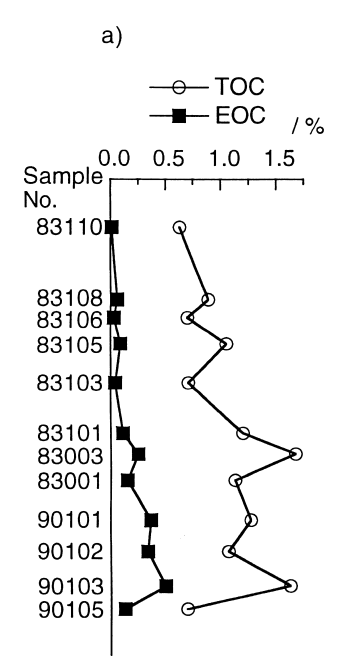

b)
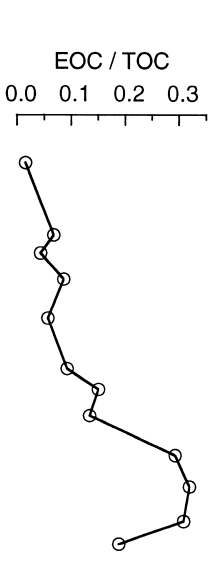

The joint study on the organic and inorganic diagenesis in this sequence revealed that petroleum formation took place mainly in the Kusanagi Formation (Taguchi et al., 1986). As the depth of sediment increases in the stratigraphic sequence, the vitrinite reflectance value increases and reaches $0.50 \%$ in the upper part of the Formation, indicating that the sediments below this part generated oil (Aiba, 1980). In accordance with the vitrinite reflectance changes, the carbon preference index (CPI) of $n$-alkanes decreases with depth and becomes close to one at the upper part of the Kusanagi Formation and that of $n$-fatty acids does at the middle part of the Kusanagi Formation (Taguchi et al., 1986). $T_{\max }$ value, temperature of maximum hydrocarbon generation from kerogen by Rock-Eval pyrolysis, is about $427^{\circ} \mathrm{C}$ at the upper Kusanagi Formation which indicates oil generation at this part of the Formation. A $T_{\max }$ value of about $427^{\circ} \mathrm{C}$ is considered on to the threshold of intense oil generation for Japanese source rocks (Connan, 1974; Kaneko et al., 1983). The stratigraphic profile of EOC/TOC shows oil generation at the upper to middle Kusanagi Formation. The stable carbon isotope study revealed

c)

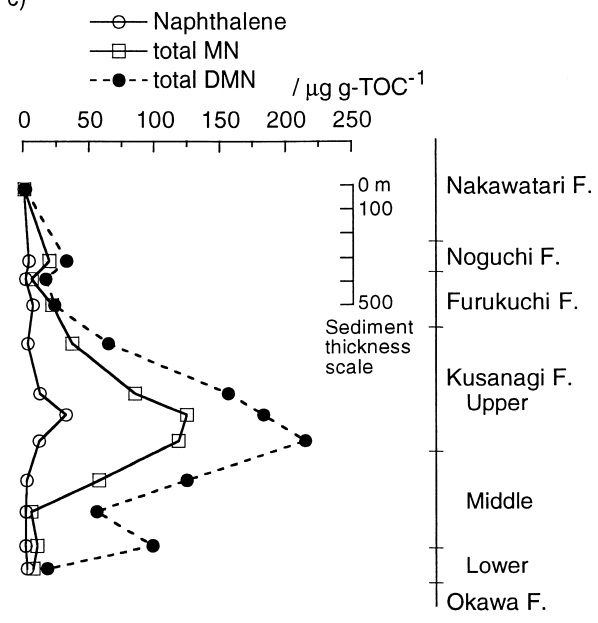

Fig. 2. Contents of total organic carbon and extractable organic compounds $(a)$, their ratios ( $b$ ), and naphthalenes (c) vs. depth in the stratigraphic sequence of the Neogene Shinjo sediments. Total organic carbon and extractable organic compounds data and their ratios are from Shimoyama and Matsubaya (1985). Abbreviations: TOC, total organic carbon; EOC, extractable organic compounds; MN, methylnaphthalene; DMN, dimethylnaphthalene. 
that the oil component was indigenous to its horizon and had been derived from kerogen at the same horizon (Shimoyama and Matsubaya, 1985). The depth profiles of the already reported compounds, such as hydrocarbons, fatty acids, steranes and triterpanes (Taguchi et al., 1986), are in accordance with those of TOC.

Since precise concentration of naphthalenes in crude oils and sediments has rarely been determined, maturation effects on concentration of naphthalenes remained unexplored. Naphthalenes in the Shinjo sedimentary sequence are principally distributed in the Kusanagi Formation as seen in Fig. 2. The depth profile of naphthalenes shows the most abundant concentration at the sample 83003 which locates at the upper part of the oil generating zone, but is not accordant with those of TOC and EOC. A marked increase of EOC/TOC ratio occurs in the region of the sample 83001 as seen in Fig. 2(b), where the threshold of intense oil generation is inferred to take place (Taguchi et al., 1986). The concentration of naphthalenes decreases in the sediment region over the sample 90101 to 90103 (Fig. 2(c)). The profile is worth noting, because concentrations of steranes and triterpanes, ones of proposed precursors of naphthalenes, generally increase with depth except for the bottom sample in this sequence (Taguchi et al., 1986). The decrease of naphthalene concentrations in the sediments of a high EOC/TOC ratio might be related to the oil generating event (heat). The heat applied to EOC in the sediments could contribute to removing volatile EOC components including rather volatile naphthalenes, although this is supported only by the fact that more volatile naphthalene and methylnaphthalenes decrease faster in this region compared to dimethylnaphthalenes as seen in Fig. 2(c). The heat could also cause decomposition of naphthalenes or their irreversible adsorption in the mineral matrix, as Burkow et al. (1990) pointed out on the basis of heating experiments of naphthalenes in the presence of clay minerals.

\section{Diagenesis}

The concentration ratios of 2- to 1methylnaphthalene found in the Shinjo sediments are plotted against the depth of the samples in Fig. 3(a). The ratio varies from 0.7 to 1.4 and shows no depth dependence. The methylnaphthalene parameter has been reported to be around one in coals or sediments with the vitrinite reflectance from

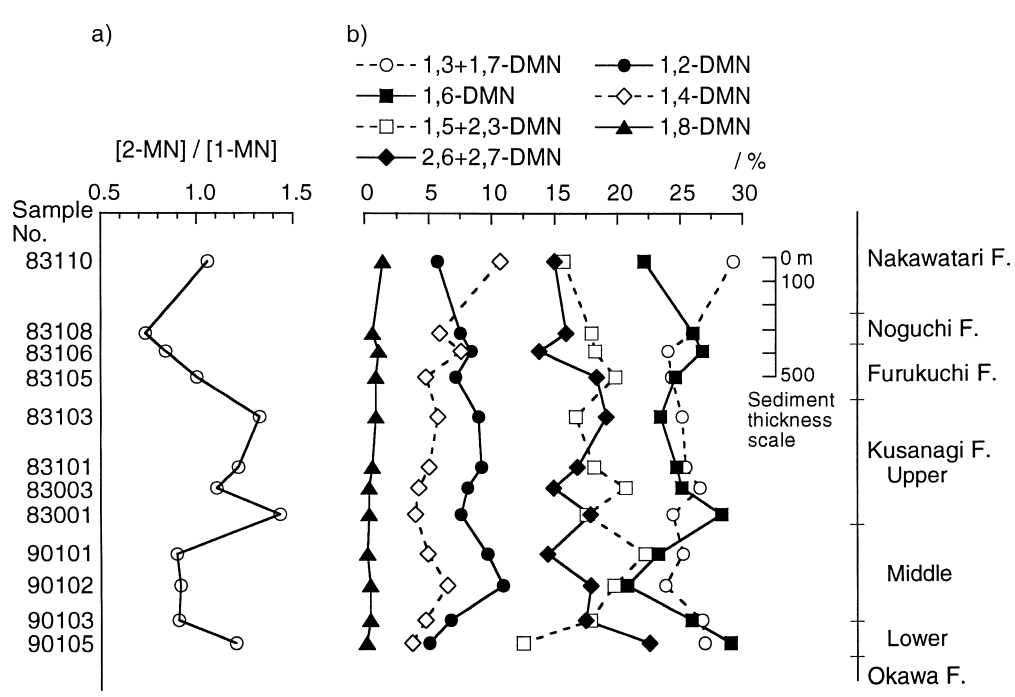

Fig. 3. Ratios of methylnaphthalenes (a) and relative abundances of dimethylnaphthalenes $(b) v s$. depth in the stratigraphic sequence of the Neogene Shinjo sediments. Abbreviations are shown in Fig. 2. 
0.5 to $1.5 \%$ and in many cases to show scattered plots against depth of samples (Radke et al., 1982a, 1984; Raymond and Murchison, 1992), being similar to our results obtained here. In our separate experiments, the samples 83106,83103 and 90103 were heated at $300^{\circ} \mathrm{C}$ for $48 \mathrm{~h}$ and the time-course of the ratio was determined by the same analytical procedure as used in this study (details of this experiment will be published elsewhere). The ratio changed gradually to a value between 2.0 and 2.5 on heating, indicating that the thermodynamically stable ratio is 2.0 or more. Such a tendency was not observed, and therefore the burial diagenesis alone had not controlled the depth profile of the concentration ratios of 2- to 1-methylnaphthalene. It might be necessary to consider the formation of methylnaphthalenes from precursors during the burial diagenesis.

The depth profiles of the relative abundances of dimethylnaphthalene isomers are shown in Fig. 3(b). No correlation is observed between the isomer abundances and the depth of the sediments, except for 1,8-isomer. In the above-stated heating experiments, the relative abundances of 2,6- and 2,7-isomer pair and 1,6-isomer changed largely after heating, compared to those of the other iso- mers, to give the constant values of $40 \%$ and $15 \%$, respectively, of the total isomers. Judging from the results, these stable isomers in the sediments seem not to be affected by the burial diagenesis. On the other hand, since the percentage of the least stable 1,8-isomer showed a decreasing trend with increasing depth, a parameter using the abundance of this isomer is likely applicable to maturity assessment in the sediments. Figure 4(a) shows plots of the negative logarithm of the 1,8-isomer abundance ratio (Alexander et al., 1984) against depth. This parameter is generally dependent on the depth of the sediments. The stratigraphic profile of this parameter is in accordance with those of the other maturity parameters, such as the vitrinite reflectance (Fig. 4(b)), Rock-Eval $T_{\max }$ (Fig. 4(c)) and the ratios of $20 \mathrm{~S}$ - to $20 \mathrm{R}$-steranes and of $22 \mathrm{~S}$ - to 22R-triterpanes (Fig. 4(d)) (Taguchi et al., 1986). The carbon preference indeces (CPI) of $n$-alkanes in the EOC and kerogen-bound fatty acids (Fig. 4(e)) decrease with increasing depth and reach minimum values in the oil-generating zone (Taguchi et al., 1986). Within the depth region where the CPIs are applicable, the 1,8dimethylnaphthalene ratio gives a good correlation with these values, but shows some scatter in

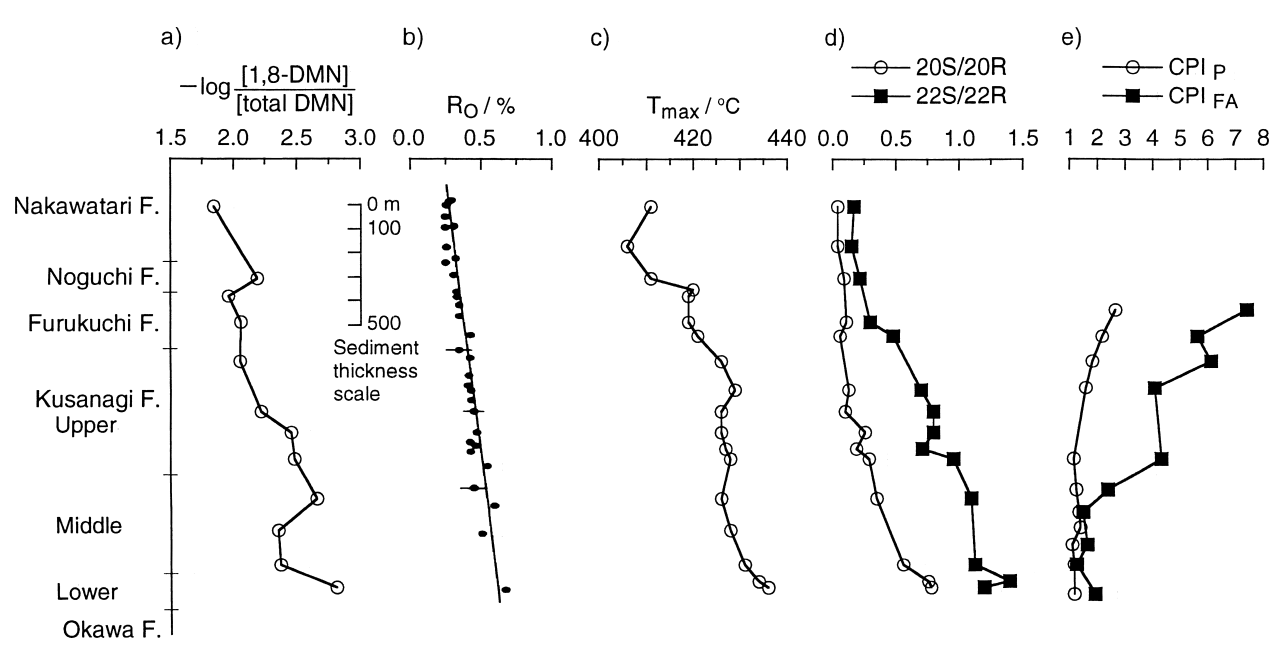

Fig. 4. Variation with depth of relative abundance of 1,8-dimethylnaphthalene (a), vitrinite reflectance (b), temperature of maximum pyrolytic yield (c), ratios of $20 S$ - to $20 R$-sterane and of $22 S$ - to $22 R$-triterpane (d), and Carbon Preference Indeces of $n$-alkanes and $n$-fatty acids (e). The data except relative abundance of 1,8 dimethylnaphthalene are from Taguchi et al. (1986). Abbreviations are shown in Fig. 2. 
the region of a high EOC/TOC ratio near the bottom sample. Although Alexander et al. (1984) had emphasized a potential use of this parameter for high maturity zones, a good correlation of 1,8dimethylnaphthalene parameter was demonstrated with the widely-accepted maturity parameters around and above the oil-generating zone of the Shinjo sedimentary sequence. Thus, the present study provides an important new example of application of the indicator to an immature zone where other maturity parameters based on isomer ratio of alkylated naphthalenes are often affected by their precursor types (Radke et al., 1984).

\section{Conclusions}

Twelve sediments from the Shinjo basin were analyzed for naphthalene and its methyl homologs through the experimental procedure that minimized their evaporative losses. The following conclusions were found to characterize naphthalenes in the Neogene sediments.

1. The total concentrations of naphthalene and its monomethyl and dimethyl homologs varied from 26 to $5,700 \mathrm{ng} \mathrm{g}^{-1}$ sample and the thermodynamically least stable compound of these, 1,8dimethylnaphthalene, was also detected in all the samples analyzed at the concentration level of 0.2 to $11 \mathrm{ng} \mathrm{g}^{-1}$.

2. Naphthalenes were principally distributed in the Kusanagi Formation. Their depth profile was not in accordance with those of TOC and EOC and showed a characteristic feature, indicating an abundance peak at the upper part of the oil-generating zone.

3. The ratio of 2- to 1-methylnaphthalene of the sample varied from 0.7 to 1.4 and showed no depth dependence.

4. No correlation was observed between the relative abundance of dimethylnaphthalene isomers and the depth of the samples, except for 1,8isomer.

5. The negative logarithm of the relative abundance of 1,8-dimethylnaphthalene showed an increasing trend with increasing depth of the sample and the depth profile was in accordance with those of the other maturity parameters.

As judged from the findings 3 and 4, no evidence was found in support of burial diagenesis which causes significant isomerization of the stable or rather stable isomers of methyl- and dimethylnaphthalene. On the other hand, the stratigraphic profile of the relative abundance of the least stable 1,8-dimethylnaphthalene isomer showed an effect of the burial diagenesis.

\section{REFERENCES}

Aiba, J. (1980) Timing of hydrocarbon migration in the main oil and gas fields in the Northeast Japan. Journal of Japanese Association of Petroleum Technologist 45, 373-382 (in Japanese).

Alexander, R., Kagi, R. and Sheppard, P. (1984) 1,8Dimethylnaphthalene as an indicator of petroleum maturity. Nature 308, 442-443.

Alexander, R., Kagi, R. I., Rowland, S. J., Sheppard, P. N. and Chirila, T. V. (1985) The effects of thermal maturity on distributions of dimethylnaphthalenes and trimethylnaphthalenes in some Ancient sediments and petroleums. Geochim. Cosmochim. Acta 49, 385395.

Burkow, I. C., Jørgensen, E., Meyer, T., Rekdal, Ø. and Sydnes, L. K. (1990) Experimental simulation of chemical transformations of aromatic compounds in sediments. Org. Geochem. 15, 101-108.

Chaffee, A. L., Strachan, M. G. and Johns, R. B. (1984) Polycyclic aromatic hydrocarbons in Australian components from Victorian brown coal. Geochim. Cosmochim. Acta 48, 2037-2043.

Connan, J. (1974) Time-temperature relation in oil genesis. American Association of Petroleum Geologists Bulletin 58, 2516-2521.

Hayatsu, R., Botto, R. E., Scott, R. G., McBeth, R. L. and Winans, R. E. (1987) Thermal catalytic transformation of pentacyclic triterpenoids: Alteration of geochemical fossils during coalification. Org. Geochem. 11, 245-250.

Kaneko, M., Taguchi, K. and Asakawa, T. (1983) Comments on pyrolytic analysis using ROCK-EVAL and its study case in Niigata Oil Field. Journal of Japanese Association of Petroleum Technologist 48, 319 (in Japanese).

Mita, H., Shigematsu, R. and Shimoyama, A. (1996) Characterization of water-extractable dicarboxylic acids in Neogene sediments of the Shinjo basin, Japan. Geochem. J. 30, 251-260.

Püttmann, W. and Villar, H. (1987) Occurrence and geochemical significance of $1,2,5,6$ - 
tetramethylnaphthalene. Geochim. Cosmochim. Acta 51, 3023-3029.

Radke, M., Willsch, H. and Leythaeuser, D. (1982a) Aromatic components of coal: relation of distribution pattern to rank. Geochim. Cosmochim. Acta 46, 1831-1848.

Radke, M., Welte, D. H. and Willsch, H. (1982b) Geochemical study on a well in the Western Canada Basin: relation of the aromatic distribution pattern to maturity of organic matter. Geochim. Cosmochim. Acta 46, 1-10.

Radke, M., Leythaeuser, D. and Teichmüller, M. (1984) Relationship between rank and composition of aromatic hydrocarbons for coals of different origins. Org. Geochem. 6, 423-430.

Radke, M., Rulkötter, J. and Vriend, S. P. (1994) Distribution of naphthalenes in crude oils from the Java Sea: Source and maturation effects. Geochim. Cosmochim. Acta 58, 3675-3689.

Raymond, A. C. and Murchison, D. G. (1992) Effect of igneous activity on molecular-maturation indices in different types of organic matter. Org. Geochem. 18, $725-735$.
Shimoyama, A. and Matsubaya, O. (1985) Stable carbon isotopes of organic matter in Neogene sediments of the Shinjo basin. Geochem. J. 19, 175-179.

Shimoyama, A., Hagishita, S. and Harada, K. (1988) Purines and pyrimidines in Neogene sediments of the Shinjo basin. Geochem. J. 22, 143-148.

Shimoyama, A., Komiya, M. and Harada, K. (1991) Low molecular weight monocarboxylic acids and $\gamma$ lactones in Neogene sediments of the Shinjo basin. Geochem. J. 25, 421-428.

Smith, J. W. and Batts, B. D. (1992) Thermal reactions of 1,8-dimethylnaphthalene. Org. Geochem. 5, 737744.

Strachan, M. G., Alexander, R. and Kagi, R. I. (1988) Trimethylnaphthalenes in crude oils and sediments: Effects of source and maturity. Geochim. Cosmochim. Acta 52, 1255-1264.

Taguchi, K., Shimoda, S., Itihara, Y., Imoto, N., Ishiwatari, R., Shimoyama, A., Akiyama, M. and Suzuki, N. (1986) Relationship of organic and inorganic diagenesis of Neogene Tertiary rocks, Northeastern Japan. Soc. Econ. Paleon. Miner. Sp. Publ. 38, 47-64. 